\title{
ANÁLISE DA DISTRIBUIÇÃO ESPACIAL DE ÁRVORES E ARBUSTOS QUANTO AO PORTE, À TAXONOMIA E À UTILIZAÇÃO ATRAVÉS DE SISTEMA DE INFORMAÇÃO GEOGRÁFICA
}

\author{
Bárbara de Oliveira Falce ${ }^{1}$; Breno Dalcolmo de Almeida Leão ${ }^{2}$, Dinorah Moraes de Souza ${ }^{3}$, \\ Fabricia Benda de Oliveira ${ }^{4}$
}

(recebido em 18.08.2011 e aceito para publicação em 15.03.2012)

\section{RESUMO}

A arborização urbana proporciona benefícios ambientais, sociais e econômicos, tornando o estudo da distribuição espacial de árvores e arbustos importante no conhecimento da qualidade de vida e bem-estar que um local oferece. O objetivo do trabalho foi identificar os componentes arbóreos e arbustivos do Centro de Ciências Agrárias da Universidade Federal do Espírito Santo e analisar a distribuição espacial destes quanto ao porte, à taxonomia e ao uso. Os pontos foram coletados com um GPS Garmim eTrexH HS e confeccionou-se mapas com auxílio do software livre TerraView 4.0.0. Foram coletados 218 pontos referentes a 22 grupos taxonômicos e foi encontrada uma densidade média de aproximadamente 54,5 plantas/ha. Fabaceae foi a família com maior freqüência relativa $(22,48 \%)$, seguida por Myrtaceae (12,39\%), Bignoneaceae e Rosaceae (10,09\%), Chrysobalanaceae (9,17\%) e Arecaceae (8,72\%). Espécimes de grande porte predominaram, a maioria acompanhando vias ou dispostas em praças e estacionamentos, e as de médio e pequeno porte compondo canteiros. Quanto à utilização, a maioria é ornamental, cuja ampla distribuição colabora para um ambiente agradável. Entretanto, observaram-se irregularidades, como frutíferas em um estacionamento e espécimes florestais próximas a prédios. Mapas de distribuição de árvores e arbustos podem fornecer subsídios para manutenção e adequação da arborização do campus.

Palavras-chave: Arborização Urbana; GPS; Geoprocessamento.

${ }^{1}$ Graduanda em Agronomia, CCA/UFES, Alto Universitário, s/noㅡ, Caixa Postal 16, Guararema 29500-000 - Alegre/ES, barbarafalce@gmail.com

2 Graduando em Agronomia, CCA/UFES, Alto Universitário, s/no, Caixa Postal 16, Guararema 29500-000 - Alegre/ES, leaobda@gmail.com

${ }^{3}$ Graduanda em Agronomia, CCA/UFES, Alto Universitário, s/noำ, Caixa Postal 16, Guararema 29500-000 - Alegre/ES, dinorah_moraes@hotmail.com

${ }^{4}$ Eng ${ }^{\mathrm{a}}$ Agrimensora, M.Sc em Eng ${ }^{\mathrm{a}}$ Civil, Professora Assistente e Doutoranda em Produção Vegetal CCA/UFES, Alto Universitário, s/nº, Caixa Postal 16, Guararema - 29500-000 - Alegre/ES,

fabriciabenda@gmail.com

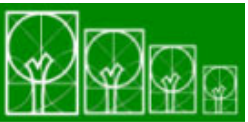

$\mathbf{S} \cdot \mathbf{B} \cdot \mathbf{A} \cdot \mathbf{U}$ Soc. Bras. de Arborização Urbana 


\section{SPATIAL DISTRIBUTION OF TREES AND BUSHES ANALYSIS BY THE SIZE, TAXONOMY AND USE THROUGH GEOGRAPHIC INFORMATION SYSTEM}

\section{ABSTRACT}

Urban forests provide environmental, social and economic benefits, making the study of trees and bushes spatial distribution important to know the life quality and welfare a place can provide. The objective of this work was to identify trees and bushes on the Agricultural Science Center of Espírito Santo Federal University and analyze the spatial distribution by the size, taxonomy and use. The points were collected with a GPS Garmin eTrex H HS and maps were made using TerraView 4.0.0 free software. 218 points were collected, related to 22 taxonomic groups and a mean density of approximately 54,5 plants/ha. Fabaceae was the family with greater relative frequency (22,48\%), followed by Myrtaceae (12,39\%), Bignoneaceae and Rosaceae (10,09\%), Chrysobalanaceae (9,17\%) e Arecaceae (8,72\%). Large-sized specimens predominated, mostly following streets or in squares and parking area, and medium and small-sized were placed in plant beds. As for the use, mostly were ornamental, which wide distribution contributes to a pleasant environment. However, some irregularities were detected, like fruit trees in a parking lot and forest trees near buildings. Maps of trees and bushes distribution can give subsidies to maintenance and adequacy for campus urban forest.

Keywords: Urban Forest; GPS; Geoprocessing.

\section{INTRODUÇÃO}

Alegre, localizada na região do Caparaó do Estado do Espírito Santo, é conhecida como Cidade Jardim devido às suas belezas naturais e ao grande número de praças ornamentadas com plantas. Entretanto, poucas informações estão disponíveis a respeito da distribuição e caracterização de plantas no município, e conhecimentos sobre a composição vegetal possibilitariam a maior valorização dos espécimes vegetais por parte dos moradores da região e despertaria maior interesse de outras pessoas envolvidas com o assunto, tais como pesquisadores.

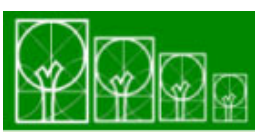

$\mathbf{S} \cdot \mathbf{B} \cdot \mathbf{A} \cdot \mathbf{U}$ Soc. Bras. de Arborização Urbana 
O Centro de Ciências Agrárias da Universidade Federal do Espírito Santo (CCAUFES), localizado em Alegre, atualmente oferece 17 cursos de graduação e 4 de pósgraduação. Apesar de alguns destes cursos não estarem diretamente relacionados com as ciências agrárias, a origem do campus está estritamente vinculada com o interesse na área. Muitos destes cursos apresentam em suas ementas disciplinas que envolvem 0 conhecimento sobre os vegetais, tais como Anatomia Vegetal, Organografia e Sistemática Vegetal, Ecologia, Paisagismo e Plantas Ornamentais, Fisiologia Vegetal e Anatomia da Madeira. A importância do conhecimento da taxonomia, utilização e porte dos exemplares vegetais do campus não se justifica apenas para o uso em cada uma destas disciplinas, mas também para permitir maior valorização e compreensão do meio neste espaço universitário.

Existem muitos trabalhos de levantamento da composição vegetal em universidades, tais como os realizados por Zuany et al. (2007) e Castro et al. (2011), sendo também amplamente discutida a atuação de universidades na educação ambiental e disseminação de conhecimentos botânicos na comunidade. A utilização do GPS para levantamento florístico é uma prática que vem conquistando espaço, devido às altas precisões que alguns aparelhos oferecem, assim como a facilidade de uso destes equipamentos e a praticidade do georreferenciamento com uso de softwares, muito dos quais são livres, facilitando o manuseio e a interpretação dos dados.

No Centro Universitário Norte do Espírito Santo (CEUNES), em São Mateus, há um projeto de arborização, e já foram plantadas mais de 800 mudas que serão utilizadas didaticamente nas aulas de Botânica e Ecologia, além de contribuir na manutenção de alguns aspectos ecológicos e contribuir para a formação de locais de visitação pública, principalmente as praças, sendo este mesmo procedimento adotado no campus de Goiabeiras, em Vitória (INFORMA, 2010). Porém, não existem informações divulgadas sobre a arborização do CCA-UFES, nem há previsão de implantação organizada de representantes vegetais no campus, o que não é compatível por este oferecer cursos relacionados e estar localizado em uma cidade denominada "Cidade Jardim".

Dessa forma, o objetivo deste trabalho foi obter informações sobre a composição arbórea e arbustiva do campus do CCA-UFES e realizar análises da distribuição espacial desses vegetais quanto ao porte, à taxonomia e à utilização, verificando a organização e as possíveis irregularidades da atual arborização, com auxílio de GPS e técnicas de Sistemas de Informação Geográfica (SIG).

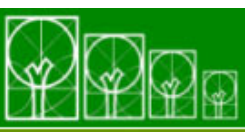

S $\cdot \mathbf{B} \cdot \mathbf{A} \cdot \mathbf{U}$ Soc. Bras. de Arborização Urbana 


\section{MATERIAIS E MÉTODOS}

O trabalho foi realizado no Centro de Ciências Agrárias da Universidade Federal do Espírito Santo, CCA-UFES (Latitude -2045’, Longitude $-41^{\circ} 32^{\prime}$ ), localizado no município de Alegre-ES (Figura 1), cujo clima de ocorrência classificado segundo Köppen, é do tipo Cwa, ou seja, tropical quente e úmido com inverno frio e seco, temperatura anual média de cerca de $23^{\circ} \mathrm{C}$ e precipitação total anual média de aproximadamente $1300 \mathrm{~mm}$ (LIMA et al., 2008).

Figura 1. Mapa de localização do Centro de Ciências Agrárias da UFES

Figure 1. Localization Map of Agricultural Science Center of UFES

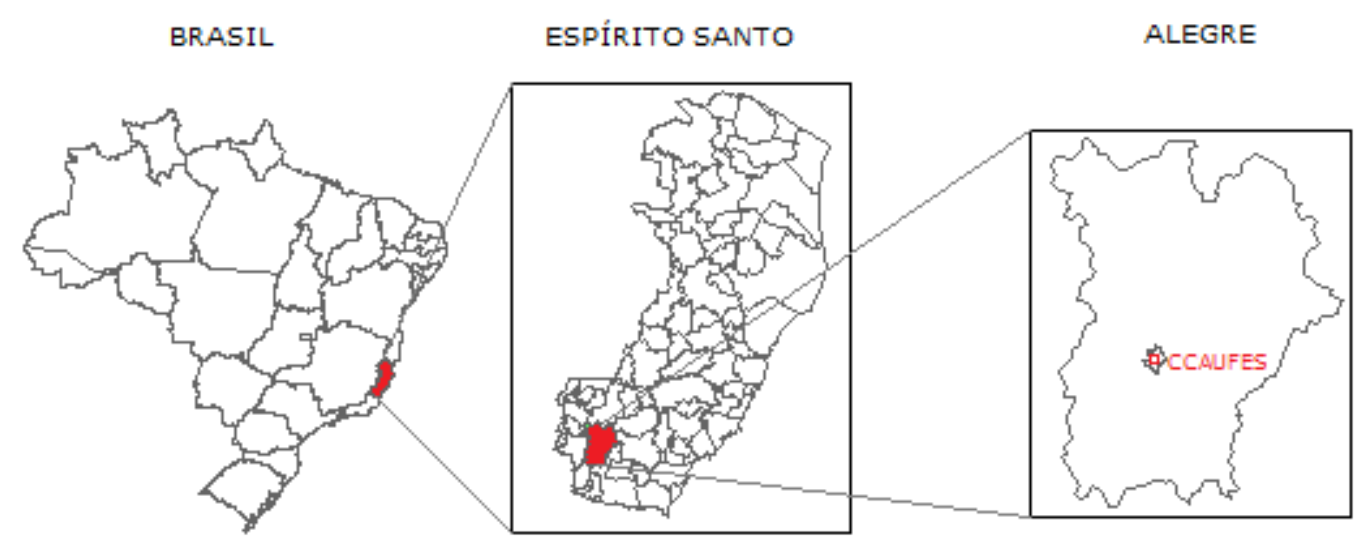

A área do CCA-UFES foi dividida em 4 subáreas, segundo a Figura 2, de forma a facilitar o processo de coleta de pontos e evitar pontos repetidos. 
Figura 2. Esquema de divisão da área do CCA-UFES em 4 subáreas para a coleta de pontos com o GPS

Figure 2. Division scheme of CCA-UFES area in four subareas used to collect GPS points

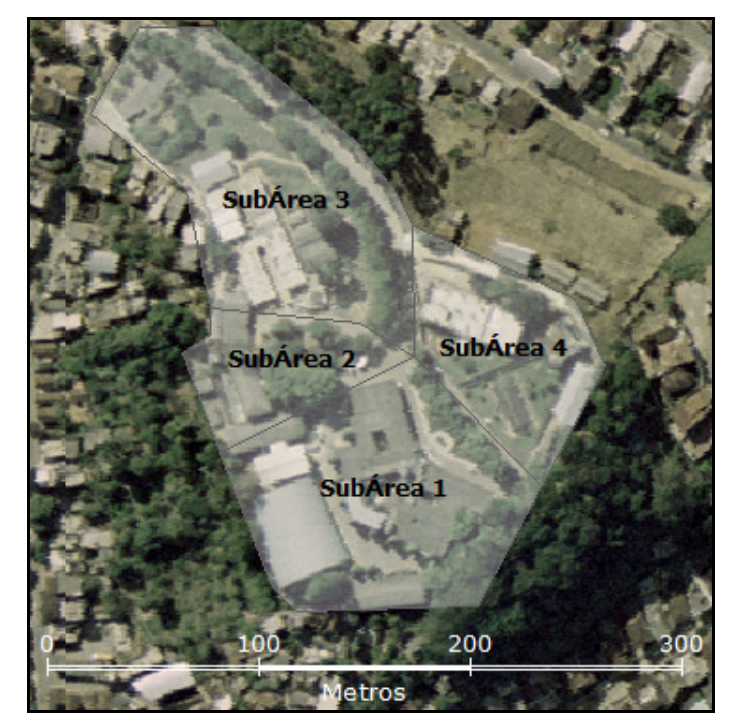

Fonte: Adaptado pelo Autor de IEMA (2007)

A coleta dos pontos foi realizada em todas as 4 subáreas com auxílio de um GPS Garmim eTrex H High Sensitivity, configurado no sistema de projeção UTM datum WGS84. Como critérios para a coleta, foram consideradas somente plantas arbóreas e arbustivas, dada a sua maior longevidade e predominância (NOBLICK et al., 1983), que não se encontravam em vasos, dentro de canteiros de obras ou de prédios. Após a coleta de cada ponto, foram anotadas informações de porte, taxonomia e utilização referentes à planta de acordo com a Tabela 1. Os critérios adotados quanto ao porte seguiram o apresentado por Mascaró e Mascaró (2005).

Tabela 1. Critérios de classificação das plantas cujos pontos foram coletados com o GPS Table 1. Classification criteria of plants which had GPS points collected

\begin{tabular}{ccc}
\hline Porte & Taxonomia & Utilização \\
\hline Pequeno $($ até $6 \mathrm{~m})$ & Família da planta. & Ornamental \\
Médio $(6$ a $10 \mathrm{~m})$ & Se Gimnosperma, Filo. & Frutífera \\
Grande $(>10 \mathrm{~m})$ & & Florestal \\
\hline
\end{tabular}


Os pontos foram descarregados em um computador e armazenados em um arquivo shapefile (shp) utilizando-se o software livre DNR Garmin. O arquivo shapefile contendo os pontos referentes às plantas e uma ortofoto de Alegre fornecida pelo IEMA (UTM datum WGS84) foram importados para o software TerraView 4.0.0. No Plano de Informação contendo os pontos das plantas, foram criadas na tabela de atributos as colunas "Porte", "Taxonomia" e "Utilização", e a tabela foi alimentada segundo os dados obtidos das observações em campo. Novos Planos de Informação foram criados contendo polígonos aproximados dos prédios, praças e estacionamentos, e linhas representando as ruas pertencentes ao CCA-UFES, ambos procedimentos baseando-se em observações da ortofoto georreferenciada do IEMA da sede do município de Alegre. Foi também georreferenciada, baseada na ortofoto cedida pelo IEMA, uma imagem do CCA-UFES recente proveniente do Google Maps ${ }^{\circledR}$, a fim de adicionar polígonos de prédios recémconstruídos.

No software TerraView 4.0.0, foram quantificados o número de exemplares de cada família encontrada. Os pontos coletados foram separados por temas quanto ao porte, taxonomia e utilização de acordo com a classificação da Tabela 1. Na Vista Taxonomia, foram criados apenas Temas de Famílias que possuíam mais de 10 exemplares, sendo que o restante dos pontos ficou agrupado em um Tema nomeado "Outros".

\section{RESULTADOS E DISCUSSÃO}

Foram coletados ao todo 218 pontos referentes a árvores e arbustos nos limites do CCA-UFES distribuídos em 22 grupos taxonômicos, conforme apresentado na Tabela 2.

Tabela 2. Número de exemplares (QNT) e freqüência relativa (FREQ) de cada grupo taxonômico encontrado no CCA-UFES

Table 2. Number of specimens (QNT) and relative frequency (FREQ) of each taxonomic group found in CCA-UFES

\begin{tabular}{ccc|ccc}
\hline $\begin{array}{c}\text { Grupo Taxonômico } \\
\text { (Taxonomic Group) }\end{array}$ & QNT & FREQ & $\begin{array}{c}\text { Grupo Taxonômico } \\
\text { (Taxonomic Group) }\end{array}$ & QNT & FREQ \\
\hline Fabaceae & 49 & $22,48 \%$ & Melastomataceae & 6 & $2,75 \%$ \\
Myrtaceae & 27 & $12,39 \%$ & Caricaceae & 2 & $0,92 \%$ \\
Bignoniaceae & 22 & $10,09 \%$ & Meliaceae & 2 & $0,92 \%$
\end{tabular}

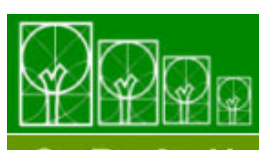

$\mathbf{S} \cdot \mathbf{B} \cdot \mathbf{A} \cdot \mathbf{U}$ Soc. Bras. de Arborização Urbana 


\begin{tabular}{ccc|ccc} 
Rosaceae & 22 & $10,09 \%$ & Solanaceae & 2 & $0,92 \%$ \\
Chrysobalanaceae & 20 & $9,17 \%$ & Agavaceae & 1 & $0,46 \%$ \\
Arecaceae & 19 & $8,72 \%$ & Annonaceae & 1 & $0,46 \%$ \\
Euphorbiaceae & 9 & $4,13 \%$ & Apocynaceae & 1 & $0,46 \%$ \\
Anacardiaceae & 8 & $3,67 \%$ & Lauraceae & 1 & $0,46 \%$ \\
Gymnosperma & 8 & $3,67 \%$ & Plumbaginaceae & 1 & $0,46 \%$ \\
Moraceae & 8 & $3,67 \%$ & Proteaceae & 1 & $0,46 \%$ \\
Malvaceae & 7 & $3,21 \%$ & Rubiaceae & 1 & $0,46 \%$ \\
\hline
\end{tabular}

A área do campus do CCA-UFES corresponde a aproximadamente 4,0 hectares, e considerados os 218 pontos coletados, têm-se uma densidade aproximada de árvores e arbustos de 54,5 espécimes/ha. Em Kurihara et al. (2005), foram encontrados 5.011 árvores em uma área de estudo de 111 hectares do campus da Universidade de Brasília, correspondendo a uma densidade de aproximadamente 45 árvores/ha. Em termos de número de grupos taxonômicos encontrados, o resultado (21 Famílias e 1 Filo) foi inferior ao de outros trabalhos realizados em campi de Universidades, onde foram encontradas 49 Famílias (KURIHARA et al, 2005), 41 Famílias (CASTRO et al., 2011), 52 Famílias (LOMBARDI e MORAIS, 2003) e 43 Famílias (RIZZO et al, 1993). Isso pode ter ocorrido pelo fato de o CCA-UFES contar apenas com uma equipe de manutenção das áreas vegetadas, deixando a desejar na produção de novos projetos de renovação e implantação de maior diversidade. Além disso, o aumento no número de cursos oferecidos demandou a construção de novos prédios ou expansão dos antigos, o que pode ter contribuído para reduzir a diversidade de Famílias.

Fabaceae constitui uma das maiores famílias de Angiospermas, sendo a principal utilizada na arborização urbana brasileira (SOUZA e LORENZI, 2008), o que corrobora com o resultado encontrado, uma vez que $22,48 \%$ das árvores e arbustos pertenciam a essa família. Dantas e Souza (2004) analisaram a arborização urbana da cidade de Campina Grande, e Fabaceae também foi o grupo taxonômico mais representativo.

Assim como os resultados observados por Lombardi e Moraes (2003), Myrtaceae, Bignoneaceae e Arecaceae representaram famílias muito expressivas na composição arbórea de um campus de Universidade. Chrysobalanaceae possui representantes comumente utilizados na arborização de praças, parques e ruas, como o Oiti (SOUZA e LORENZI, 2008), e Rosaceae é amplamente utilizada na ornamentação de canteiros, justificando a freqüência relativa significativa destas famílias no campus do CCA-UFES.

A distribuição quanto ao porte pode ser observada na Figura 3.

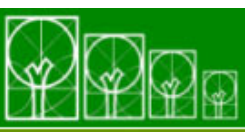

$\mathbf{S} \cdot \mathbf{B} \cdot \mathbf{A} \cdot \mathbf{U}$ Soc. Bras. de Arborização Urbana 
Figura 3. Mapa da distribuição de árvores e arbustos no CCA-UFES quanto ao porte

Figure 3. Distribution Map of trees and bushes in CCA-UFES by the size

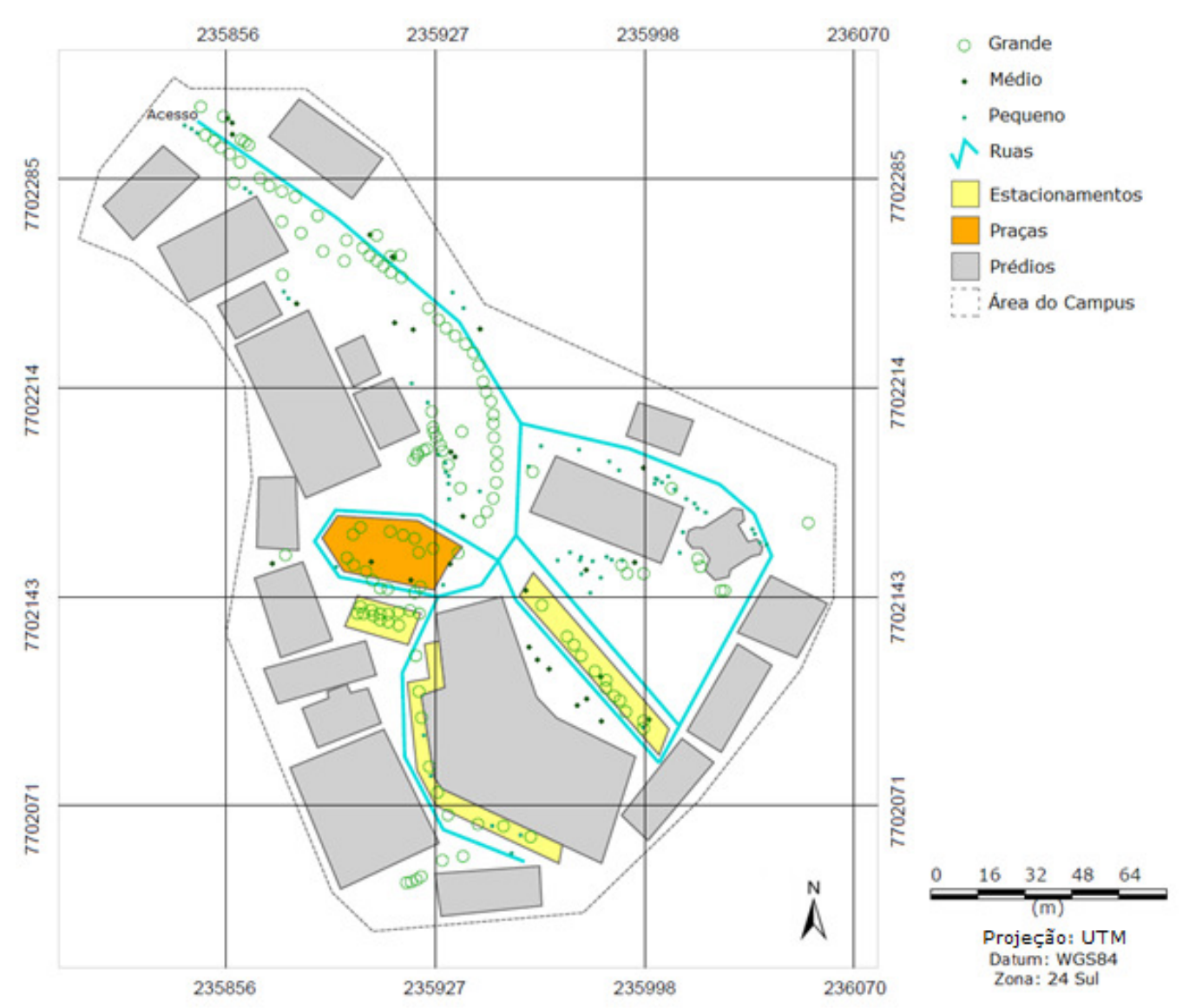

Observa-se a predominância de espécimes de grande porte, sendo que algumas destas plantas estão dispostas de forma desorganizada, enquanto outras provavelmente seguiram um planejamento de implantação. Estas plantas dispostas de forma organizada não apenas acompanham a ladeira de acesso a partir da entrada do campus, como também se encontram na região da praça e nos estacionamentos. Considerando-se apenas o grande porte, estas árvores são desejáveis em tais locais, devido à sombra que proporcionam (SANTANA e SANTOS, 1999), colaborando com a redução da radiação solar direta nos veículos dos estacionamentos, e amenizando temperaturas tanto na ladeira de acesso quanto na praça, que representa local de encontro principalmente de estudantes. Observase ainda que os vegetais de porte médio e os de pequeno porte (categorias menos representativas) encontram-se praticamente no entorno dos prédios, formando canteiros.

A Figura 4 apresenta a distribuição das árvores e arbustos quanto à taxonomia.

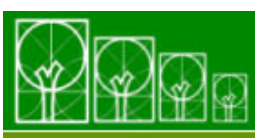

$\mathbf{S} \cdot \mathbf{B} \cdot \mathbf{A} \cdot \mathbf{U}$ Soc. Bras. de Arborização Urbana 
Figura 4. Mapa da distribuição de árvores e arbustos no CCA-UFES quanto à taxonomia

Figure 4. Distribution Map of trees and bushes in CCA-UFES by the taxonomy

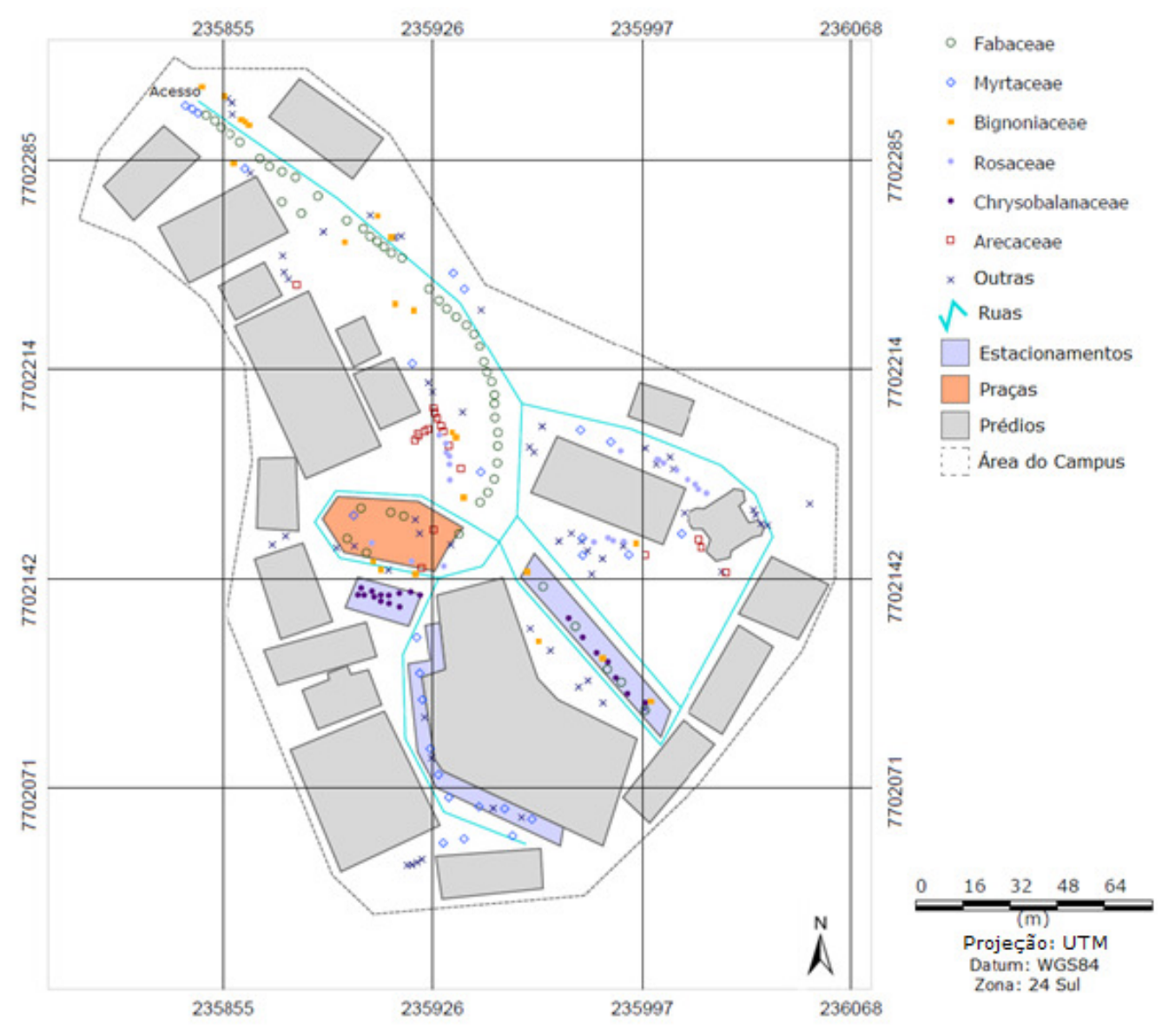

Pode-se observar na Figura 4 que Fabaceae tem representantes que acompanham principalmente ruas, enquanto que Rosaceae e Arecaceae distribuem-se praticamente em torno de prédios. Exemplares de Chrysobalanaceae só estão presentes em estacionamentos, provavelmente pela sombra que proporcionam e pelos frutos, os oitis, não representarem riscos aos veículos por seu tamanho e peso reduzido. Bignoniaceae e Myrtaceae, assim como os grupos taxonômicos enquadrados em "Outros" encontram-se bem distribuídos no campus, apesar de não haver um planejamento claro de implantação, pois os exemplares não seguem um padrão de distribuição.

A distribuição quanto à utilização das árvores e arbustos é apresentada na Figura 5. 
Figura 5. Mapa da distribuição de árvores e arbustos no CCA-UFES quanto à utilização

Figure 5. Distribution map of trees and bushes in CCA-UFES by the use

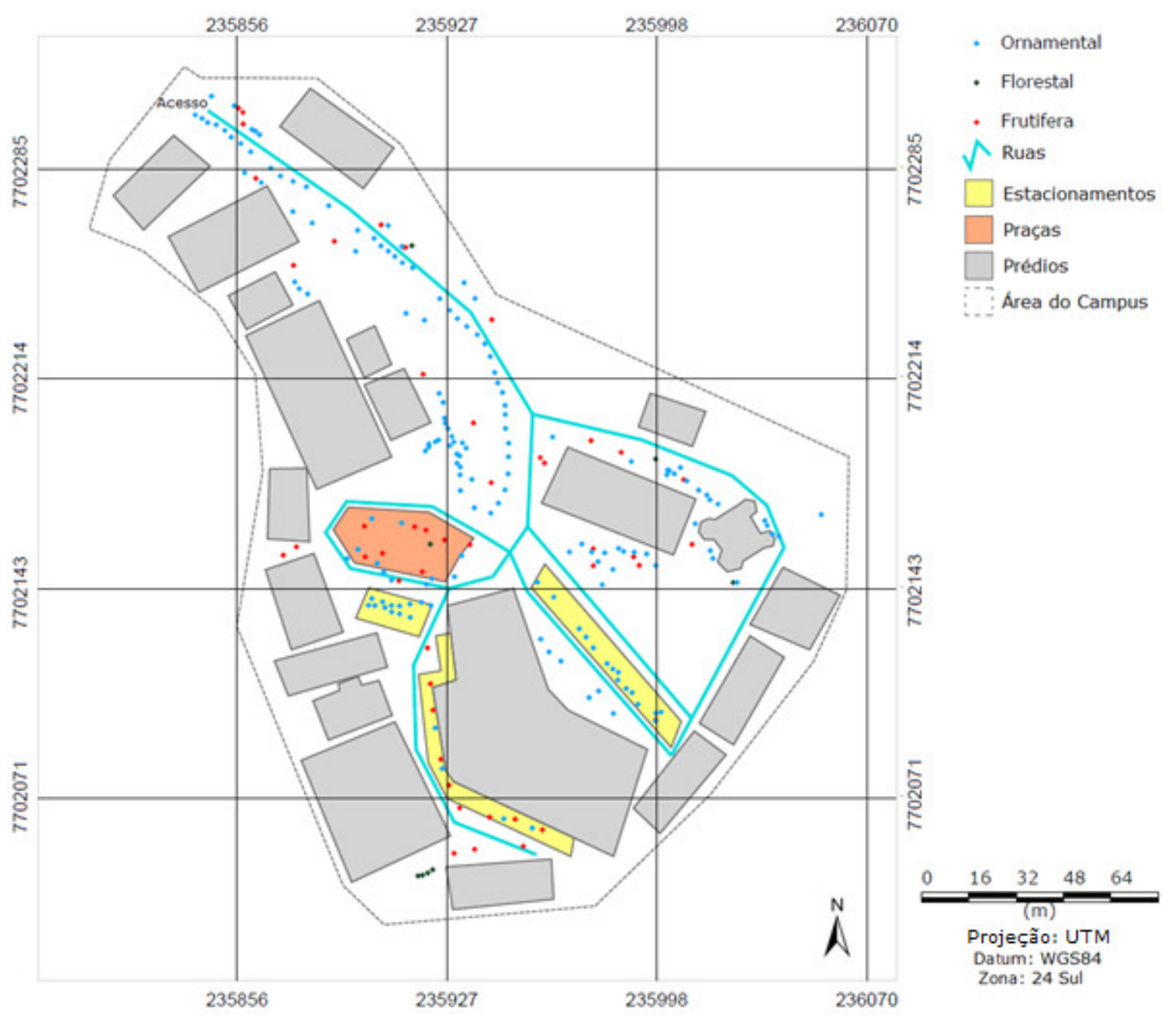

Quanto à utilização (Figura 5), pode-se observar que a maioria das plantas são ornamentais e há exemplares distribuídos praticamente por todo o CCA, fatos que colaboram com a formação de um ambiente mais belo e agradável aos freqüentadores do campus. As frutíferas representam a segunda categoria mais expressiva, sendo que o único problema quanto à sua distribuição é a presença de exemplares em um dos estacionamentos, o que pode significar riscos para os veículos pela queda de frutos. Os espécimes florestais são praticamente desprezíveis quanto ao número, e sua distribuição não é das mais criteriosas. Isto porque a maioria das espécies florestais apresenta sistema radicular extremamente agressivo, o que pode representar riscos aos sistemas de encanamento subterrâneo, ao calçamento e às construções próximas. 


\section{CONCLUSÕES}

Comparado a outros campi de universidades brasileiras, o CCA-UFES apresentou relativa pobreza em número de Famílias da composição arbórea e arbustiva, todavia, o número de espécimes por hectare pode ser considerado satisfatório.

De forma geral, a distribuição de árvores e arbustos abrange boa parte da área total do campus, entretanto, pode-se observar algumas irregularidades na localização de alguns exemplares, como é o caso da presença de frutíferas em um dos estacionamentos e a de espécies florestais em locais inadequados.

A confecção de mapas temáticos da distribuição espacial dos vegetais com uso de GPS e técnicas de geoprocessamento possibilita, muito além da apresentação de dados da composição arbórea e arbustiva, a identificação da organização e das irregularidades de forma eficiente, fornecendo subsídios importantes para a manutenção e a adequação da arborização no campus.

\section{REFERÊNCIAS}

CASTRO, A. S. F.; MORO, M. F.; ROCHA, F. C. L. Plantas dos espaços livres da Reitoria da Universidade de Fortaleza (UNIFOR), Ceará, Brasil. Revista Brasileira de Biociências, Porto Alegre, v.9, n. 1, p. 126-129, jan./mar. 2011.

DANTAS, I. C.; SOUZA, C. M. C. Arborização Urbana na cidade de Campina Grande - PB: Inventário e suas espécies. Revista de Biologia e Ciências da Terra, Campina Grande, v.4, n.2, 2004.

IEMA - INSTITUTO ESTADUAL DE MEIO AMBIENTE. Acervo de fotos digitais ortorretificadas do Aerolevantamento do Estado do Espírito Santo - GEOBASES. 2007. Disponível em: <http://200.140.47.75/geobasesonline/mapa.html>. Acesso em: 16 de jun. 2011.

INFORMA. Informativo da Universidade Federal do Espírito Santo n 348.14 a 20 de junho de 2010.

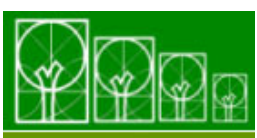

$\mathbf{S} \cdot \mathbf{B} \cdot \mathbf{A} \cdot \mathbf{U}$ Soc. Bras. de Arborização Urbana 
KURIHARA, D. L.; IMANÃ-ENCINAS, J.; PAULA, J. E. Levantamento da arborização do campus da Universidade de Brasília. Cerne, Lavras, v.11, n.2, p. 127-136, abr./jun. 2005.

LIMA, J. S. S.; SILVA, S. A.; OLIVEIRA, R. B.; CECÍLIO, R. A.; XAVIER, A. C. Variabilidade temporal da precipitação mensal em Alegre-ES. Revista Ciência Agronômica, Fortaleza, v.39, n.2, p.327-332, 2008.

LOMBARDI, J. A.; MORAIS, P. O. Levantamento florístico das plantas empregadas na arborização do campus da Universidade Federal de Minas Gerais, Belo Horizonte-MG. Lundiana, Belo Horizonte, v.4, n.2, p.83-88, 2003.

MASCARÓ, L; MASCARÓ, J. Vegetação urbana. 2.ed. Porto Alegre: Mais Quatro Editora, 2005. 204 p.

NOBLICK, L. R.; BORGES, K. N., LEMOS, M. J. S. Levantamento das plantas ornamentais introduzidas no campus da Universidade Estadual de Feira de Santana. Sitientibus, Feira de Santana, v. 2, n. 3, p. 37-58, jul./dez. 1983.

RIZZO, J. A.; FILHO, J. R.; HASHIMOTO, M. Y. Estudo da arborização e das áreas verdes do campus II da Universidade Federal de Goiás. Anais Esc. Agron. e Vet., Goiás, v.23, n.1, p.19-45, jan./dez. 1993.

SANTANA, J. R. F. S., SANTOS, G. M. M. Arborização do Campus da UEFS: exemplo a ser seguido ou um grande equívoco. Sitientibus, Feira de Santana, v. 20, p. 103-107, 1999.

SOUZA, V. C.; LORENZI, H. Botânica Sistemática. Nova Odessa: Instituto Plantarum, 2008.

ZUANY, L. V.; PRATES, E. M. B.; FRANCO, M. P. M.; GALHARDO, I. C.; ALBUQUERQUE, R. W.; FRANK-DE-CARVALHO, S. M. Levantamento florístico de uma área de Cerrado da Universidade de Brasília. Revista Brasileira de Biociências, Porto Alegre, v.5, supl. 2, p. 801-803, jul. 2007. 